\title{
Design of Remote Interworking Intelligent Medicine Chest Based on MSP430 Microcontroller
}

\author{
Huang Hui ${ }^{\mathrm{a}}$, Lu Qingru ${ }^{\mathrm{b}}$, Xin Haiyan ${ }^{\mathrm{c}}, \mathrm{Zuo} \mathrm{Mei}^{\mathrm{d}}$ \\ Department of electronic engineering, Chengxian College, Southeast University, Nanjing Jiangsu 210000, \\ China \\ aemail: huanghui_1225@163.com, bemail:silver_1120@163.com, cemail: \\ 983511889@qq.com, demail:1378096673@qq.com
}

Keywords: MSP430; Smart medicine chest; Parameters detection; Remote Interworking

\begin{abstract}
Aiming at the problem of drug waste and drug management that is prevalent in social life, and at the same time making up for the shortage of medical kits on the market., this paper takes the 16-bit single-chip MSP430F4616 as the core according to the micro-power, functional and real-time design requirements, and introduces its overall design idea and concrete implementation method in detail. The whole system includes functional modules such as physiological condition monitoring, drug monitoring, and wireless communication. Compared with the traditional medicine chest, the system has the advantages of practicality, convenient and flexibility.
\end{abstract}

\section{Introduction}

At present, there is an alarming waste in the field of Chinese medicine. According to statistics, the annual waste caused by expiration of the validity period is 15,000 tons, and the value of wasted drugs is more than 10 billion yuan. There are many factors leading to drug waste, such as the lack of systematic management of household medicines, people do not have an interactive platform to share drug information in the market. On the other hand, with the development of society and the continuous improvement of people's living standards, as well as the promotion of "Internet + " and the continuous maturity and application of Internet of things, household appliances tend to be informative and intelligent, and all kinds of intelligent medical products are springing up and gradually changing people's production and daily life style. People are no longer satisfied with the basic functions of the product, the intelligent and humanized products have higher requirements. In this paper, the intelligent medicine chest combined with "Internet + " has broken the traditional management system of medicine chest, and has the functions of temperature and humidity monitoring, drug information storage, remote drug monitoring and early warning, and physiological monitoring of human body. To provide users with more detailed, accurate and humanized drug monitoring and technical services, to prevent drug shortages or excessive purchase, to establish a safer, environmental protection, reliable drug system, and ultimately solve the problem of family drug safety.

\section{The System Design}

This design mainly aims at the problem of improper household drug management, using Internet technology to design a specific reminder, monitoring, information management intelligent medicine chest. The overall functions of the intelligent medicine chest are as follows:

1) It can monitor the environmental information of the medicine chest. The storage of drugs requires a clean, dry and suitable temperature environment. High humidity easily leads to drug moldy deterioration. High humidity easily leads to drug moldy deterioration. The intelligent medicine chest can monitor the temperature and humidity environment inside the medicine chest at any time.

2) It can remind users to take medicine regularly. Smart medicine chest has a reminder function, for chronic patients, can avoid missing the best time to take medicine. 
3) It can provide drug information storage and management services.The medicine chest provides early warning of insufficient and expired drugs,and automatic classification for special drugs.

4) Smart medicine chest uses wireless network to realize remote communication with mobile terminal. Users can check the drug information in the medicine chest remotely through the mobile terminal.

The overall design framework of the system is shown in Fig 1. The system includes the main controller, temperature and humidity sensor, infrared sensor, physiological parameter detection module, mechanical control module, Wi-Fi module. The biggest feature of the system design is the remote interconnection between the medicine chest and the mobile phone through wireless network. The main process of the system is to detect the temperature and humidity information in the medicine chest by the temperature and humidity sensor, and transmit the information to the main controller, and display it on the LCD in real time to prevent the medicine from deteriorating. By measuring basic physiological state of human body such as heart rate, users can better grasp the physical condition, rational drug use. When the key physiological parameter is abnormal, the monitoring instrument should give an alarm automatically, and the user can arrange medical treatment in time. The storage status of the medicine chest is detected by infrared sensor, and the supplement of the medicine is reminded in time. In addition, by scanning the drug bar code, the obtained drug data is transmitted to the database for information storage. At the same time, the mobile phone client through APP to view drug information, drug expiration reminders and other functions, to create a safer and more environmentally friendly environment for users.

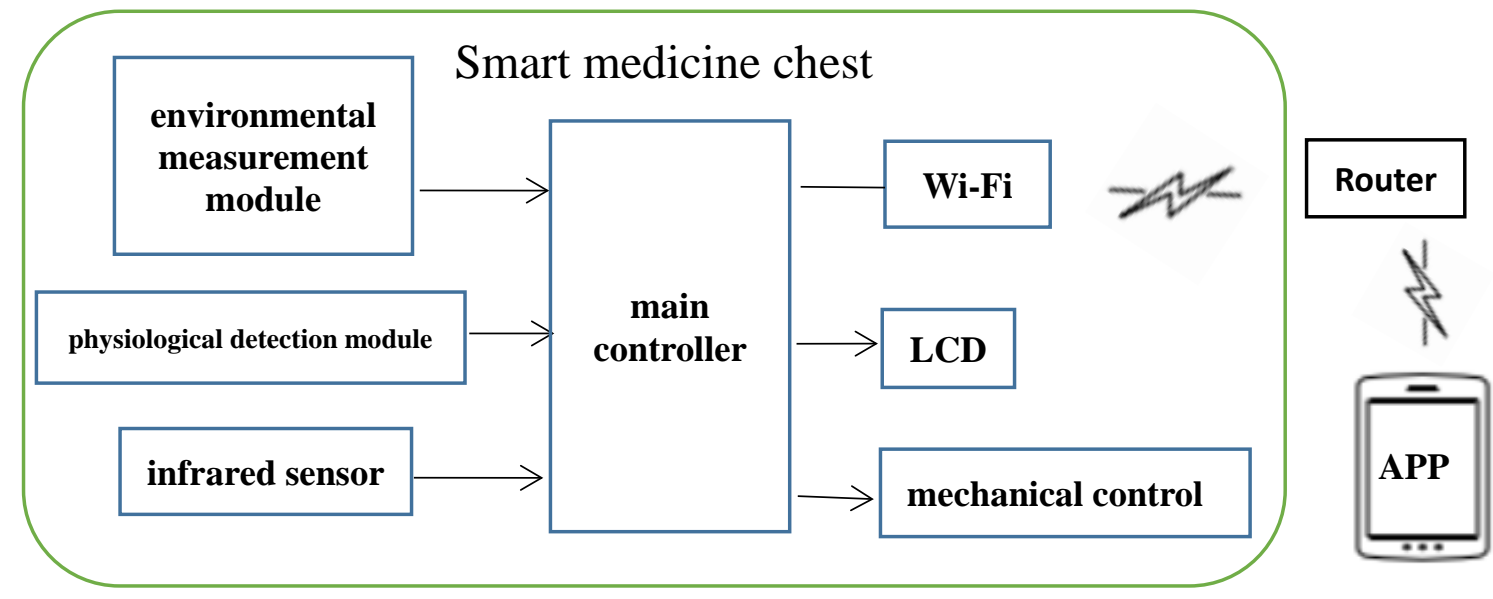

Fig 1 Schematic diagram of system structure

\section{The Hardware Platform}

The whole system is designed around the requirement of low power consumption.The system adopted MSP430F149IPM chip as the main controller. MSP430F149IPM is an ultra-low power 16 bit microcontroller designed and manufactured by TI. It has low voltage, ultra-low power consumption, fast wake-up and other characteristics, including 12-bit precision 12-channel A/D converter, 60KB flash memory, 2KBram, hardware multiplier, 2 SPI port, 2 UART port and so on. It helps improve system integration, improve micro power design

LCD 12864 chip of display module can display Chinese character menu, physiological parameter value, environmental parameter, medication reminder and medication information. The LCD module has high luminous efficiency and low voltage power supply (3.0-5.5V) and low power consumption. It receives data through serial port, and has built-in DC-DC conversion circuit. It does not need additional negative voltage, and provides a simple and convenient monitoring interface for users. d micro design.

The system uses infrared sensor to detect whether the medicine in the medicine chest is used up. The environmental measurement module in the intelligent medicine cabinet is mainly realized by the DHT11 temperature and humidity composite sensor, which has calibrated digital signal output. 
The humidity measurement precision arrives at $\pm 5 \% \mathrm{RH}$, the temperature measurement precision is $\pm 2{ }^{\circ} \mathrm{C}$, humidity measuring range is from $20 \% \mathrm{RH}$ to $90 \% \mathrm{RH}$, temperature measuring range is from $0^{\circ} \mathrm{C}$ to $50^{\circ} \mathrm{C}$.

The measurement of the human heart rate is mainly realized by the pulsesensor pulse heart rate sensor. The module determines the human heart rate according to the principle of photoelectric reflection. When the light beam passes through the peripheral blood tube of the human body, the transmittance of the light beam changes due to the change of arterial pulse congestion volume. At the same time, the light reflected by human tissue is received by the photoelectric converter and transformed into an electrical signal. The sensor outputs the analog signal and directly inputs the electrical signal to the MSP430.The MCU calculates the input signal, and by calculating the time of the peak point of two adjacent pulse waves and filtering, the time between the two heartbeats is obtained as the IBI value. By formula BPM(heart rate)=60/IBI, the heart rate value is obtained.

The system adopts ESP8266-12f wireless communication module produced by Ai-thinker company. It was embedded with TCP/ IP protocol. The communication between Wi-Fi module and MCU is through serial port for data exchange. The module can automatically convert the serial protocol to wireless and transmit data to the router or home gateway. The conversion process is shown in Fig 2.

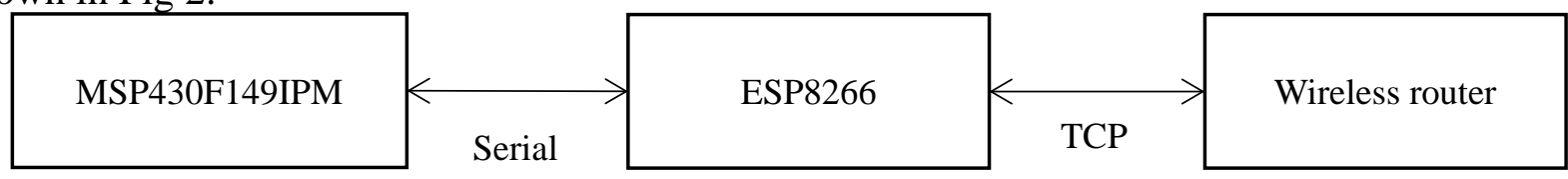

Fig.2 Transformation model diagram

\section{The Software Design}

The system needs to achieve physiological parameters data, environmental parameters data, drug chest residue data acquisition and storage, and then data display, wireless transmission. On the basis of considering the functional, real-time and reliability requirements, this design uses interrupt structure control program to achieve the above functions. Specifically, the sensor data of the physiological parameter acquisition module and the like are read through the interruption of the serial data reception, and the data is read once every cycle. All data are temporarily stored in the data buffer of RAM in the MSP430 chip. The main program flow chart of the system is shown in Fig 3. After the MCU is powered on, the system is initialized, including the chip serial port settings, memory requests, timer settings, peripheral equipment initialization and detection. Then the system starts detecting whether the connection with ESP8266 is successful.

According to the system structure diagram, the functions of APP software on Android mobile phone are divided into drug information management module, drug chest environment detection module, user personal information module and drug chest control module. Fig 4 shows the architecture of APP software function design.

The drug information management module is the most core module in the whole system. By scanning the barcode of drugs through mobile phones, the input, classification and storage of drug information can be realized. It can find out if there is a drug in the family's medicine cabinet by typing in the symptoms. It can also remind that if drugs are about to expire.

The environmental detection module of the kit is graphically rendered. It can display temperature and humidity monitoring data in real time. It can give a temperature alarm when the value exceeds the preset range.

The user personal information management module includes user login, user case information, and medication reminding function. The control module mainly realizes the remote switch control of the medicine chest. 


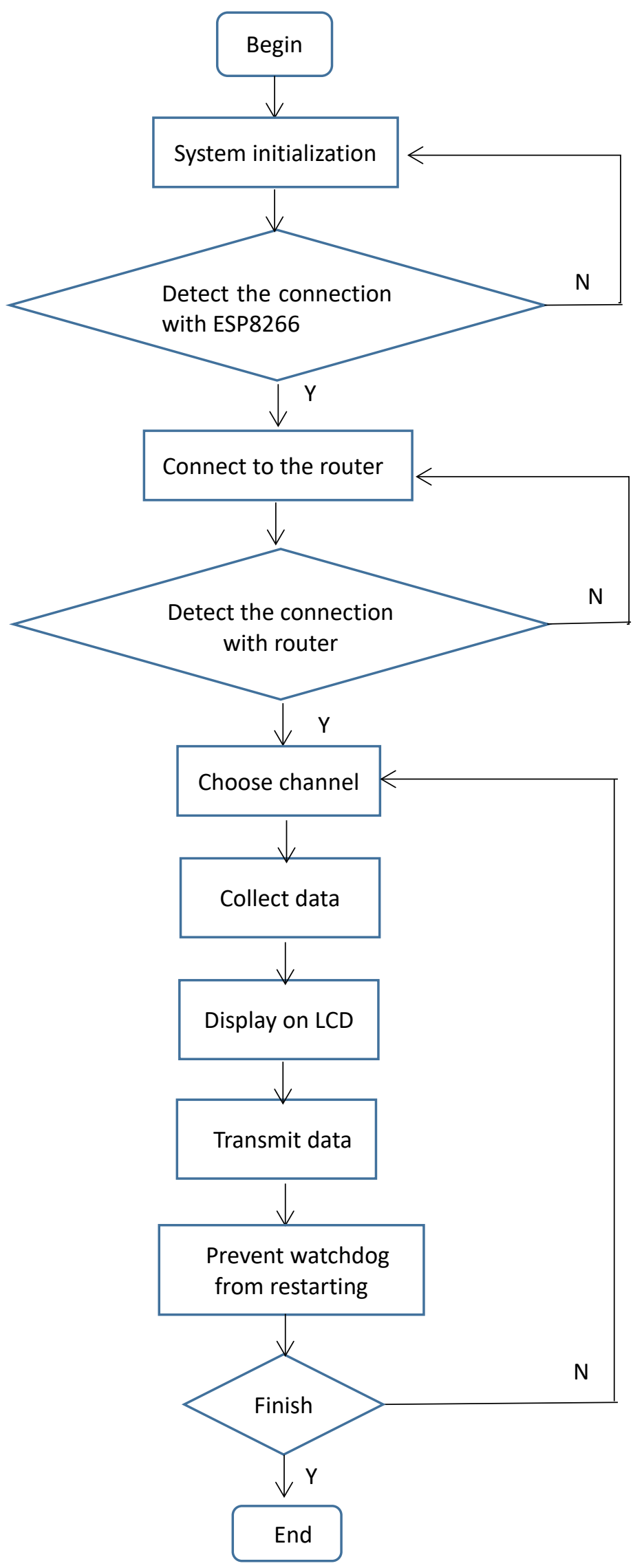

Fig 3 The main program flow chart 


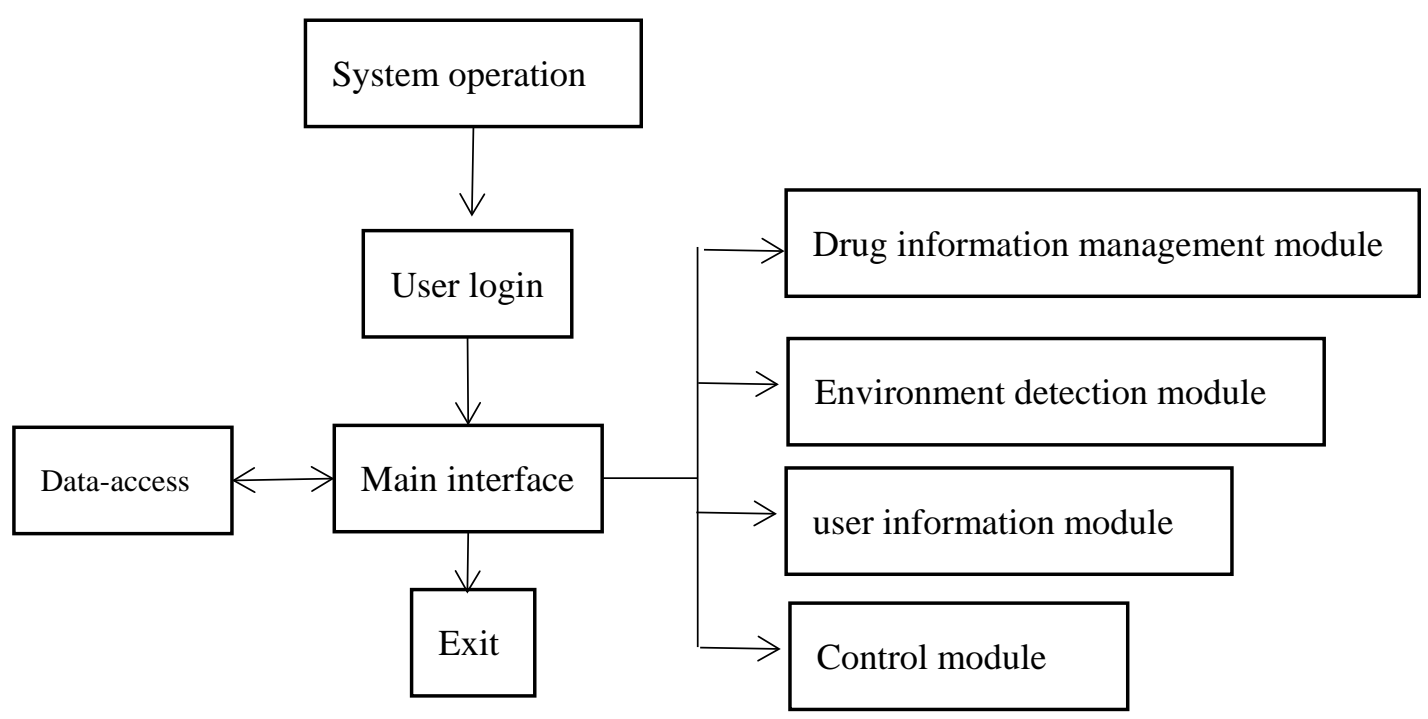

Fig 4 Function flow chart of APP software system

\section{Conclusions}

For the disadvantages of the traditional medical chest on the market and the desire for smart home, this paper designs an intelligent medical chest system based on MSP430.This intelligent medicine chest has injected a new direction for future medical and health care. "Internet $+"$ plus traditional medicine chest breaks the single management drug system, provides people with more humane and convenient service system, and creates an intelligent, multi-functional modern medicine chest product. After testing, the system runs stably, uses conveniently, combines its own scientific and intelligent characteristics, has a good application prospect.

\section{References}

[1] Botta A, Donato W D, Persico V, et al. Integration of Cloud Computing and Internet of Things: A survey [J]. Future Generation Computer Systems, 2015, 56(C): 684-700.

[2] Varshney U. Smart Medication Management System and Multiple Interventions for Medication Adherence [J]. Decision Support Systems, 2013, 55(2): 538-551.

[3] Deen M J. Information and Communications Technologies for Elderly Ubiquitous Healthcare in a Smart Home [J]. Personal and Ubiquitous Computing, 2015, 19(3): 573-599.

[4] Shin D. A Socio-Technical Framework for Internet-of-Things Design: A Human-Centered Design for the Internet of Things [J]. Telematics and Informatics, 2014, 31(4): 519-531.

[5] Jararweh Y, Al-Ayyoub M, Darabseh A, et al. SDIo T: A Software Defined Based Internet of Things Framework [J]. Journal of Ambient Intelligence and Humanized Computing, 2015, 6(4): 453-461. 